\title{
Electrical shielding of MV/MF transformers subjected to high dv/ dt PWM voltages
}

\section{Conference Paper}

\section{Author(s):}

Guillod, Thomas; Krismer, Florian; Kolar, Johann W. (D)

Publication date:

2017

Permanent link:

https://doi.org/10.3929/ethz-b-000130827

Rights / license:

In Copyright - Non-Commercial Use Permitted

Originally published in:

https://doi.org/10.1109/APEC.2017.7931050 


\title{
Electrical Shielding of MV/MF Transformers Subjected to High dv/dt PWM Voltages
}

\author{
Thomas Guillod, Florian Krismer, and Johann W. Kolar \\ Power Electronic Systems Laboratory (PES), ETH Zurich, 8092 Zurich, Switzerland
}

\begin{abstract}
The introduction of Medium-Voltage (MV) SiliconCarbide (SiC) devices enables the usage of higher power converter operating voltages, switching frequencies, and commutation speeds. This implies that Medium-Frequency (MF) and High-Frequency (HF) transients are applied to passive components, and particularly to inductors and transformers. Together with the operation at medium voltage, this leads to challenging situations with respect to Common-Mode (CM) currents, parasitic resonances, insulation coordination, and EMI. The electrical field is the key parameter for the aforementioned effects. Therefore, this paper analyzes the electric field distribution (in the insulation, at the surface, and in the air) for a $\pm 3.5 \mathrm{kV} / \pm 400 \mathrm{~V}, 50 \mathrm{kHz}, 25 \mathrm{~kW} \mathrm{MV} / \mathrm{MF}$ transformer employed in a Solid-State Transformer (SST) demonstrator. For reducing the field, a suitable shield is designed. It is found that the shield drastically reduces the field at the surface of the transformer and in the air without increasing the losses.

Index Terms-Power Electronics, Dielectrics and Electrical Insulation, Electromagnetic Shielding, Medium-Frequency Transformers, Medium-Voltage Transformers.
\end{abstract}

\section{INTRODUCTION}

Many emerging applications, such as MV connected renewable energy plants or large DC loads, require an isolated MV DC-DC conversion stage [1]-[3]. It has been shown that MV SiC switches are particularly interesting for these applications [1], [4]. However, due to the increased voltage, switching frequency, and commutation speed applied to the MV/MF transformer new challenges arise [3], [5]-[7].

The increased switching frequency has a direct impact on the core losses (eddy currents and hysteresis) and winding losses (skin and proximity effects) [3]. Additionally, the increased voltages and switching speeds lead to large CM currents [8], more intense excitations of parasitic resonances of the transformer [4], [9], and issues related to insulation coordination, such as dielectric losses or partial discharges [10], [11]. For all these effects, it appears that the magnetic and electric fields are of key importance for MV/MF transformers [12], [13].

Usually, the radiated field of power electronic converters is only measured in the $\mathrm{MHz}$ range at a distance of some meters and for the complete system [14]. The shielding of radiated fields is realized with a metallic/magnetic enclosure of the converter. However, the reactive (nonradiated) magnetic and electric fields originating from a transformer can also cause substantial challenges inside the converter. The magnetic field creates eddy currents which produce losses in metallic elements and disturbances in electronic circuitry [3], [14], [15]. The electric near-field allows for displacement currents and/or capacitively coupled voltages due to the parasitic capacitances between the transformer and surrounding metallic elements, which can also cause disturbances in control and sensing circuitry [14], [16] and insulation coordination issues for non-earthed parts, e.g. heat-sinks or fans [3]. The stray capacitances between the windings also provide high frequency conduction paths, which create and/or transmit conducted EMI across the galvanic isolation [4], [8].

Furthermore, the electric field is critical for the insulation coordination of the transformer and should be well-defined for a large frequency range (DC/MF/HF). Exceedingly high electric field values (inside the insulation or at the surface) produce partial and/or surface discharges, which damage the insulation and lead to breakdowns and flashovers [13], [17]-[19].

To the knowledge of the authors, the electric field of $\mathrm{MV} / \mathrm{MF}$ transformers has been studied inside the insulation [11], [12] but not at the surface of the insulation and in the surrounding air. Therefore, this paper analyzes the electric near-field and methods to mitigate excessive field strengths. Section II presents the considered DC-DC converter structure and the MV/MF transformer. The electric and magnetic field patterns of the transformer are analyzed in Section III. Finally, in Section IV, different shielding concepts, which reduce the electric field without increasing the losses, are presented.

\section{MV/MF TRANSFORMER}

\section{A. DC-DC Converter}

Recent literature identifies different converter topologies suitable for the realization of MV DC-DC converters, depending on particular needs, e.g. single-cell or multi-cell structures. In [11], [12], the transformer of the single-cell structure is identified as particularly critical with respect to the electric fields since one transformer has to deal with the complete CM and Differential-Mode (DM) voltages. Therefore, a single-cell series-resonant DC-DC converter, depicted in Figs. 1(a) and (b), is considered for the further analysis with the following ratings: $7 \mathrm{kV} / 400 \mathrm{~V}, 50 \mathrm{kHz}, 25 \mathrm{~kW}$.

Fig. 1(c) shows the transformer equivalent circuit. The stray inductance of the transformer, together with the series capacitor located on the Low-Voltage (LV) side, is used as a resonant tank. The converter is operated at the resonance frequency and Fig. 1(d) depicts the corresponding currents and voltages applied to the transformer. Zero Voltage Switching (ZVS) is achieved with the magnetizing current of the transformer. This implies that the switched current is nearly 


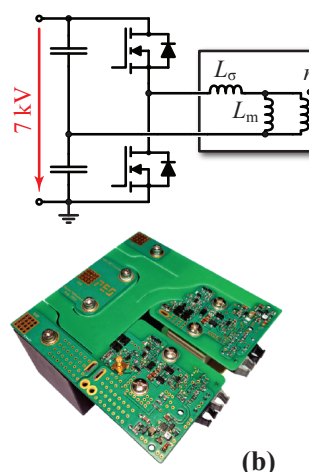

(b)
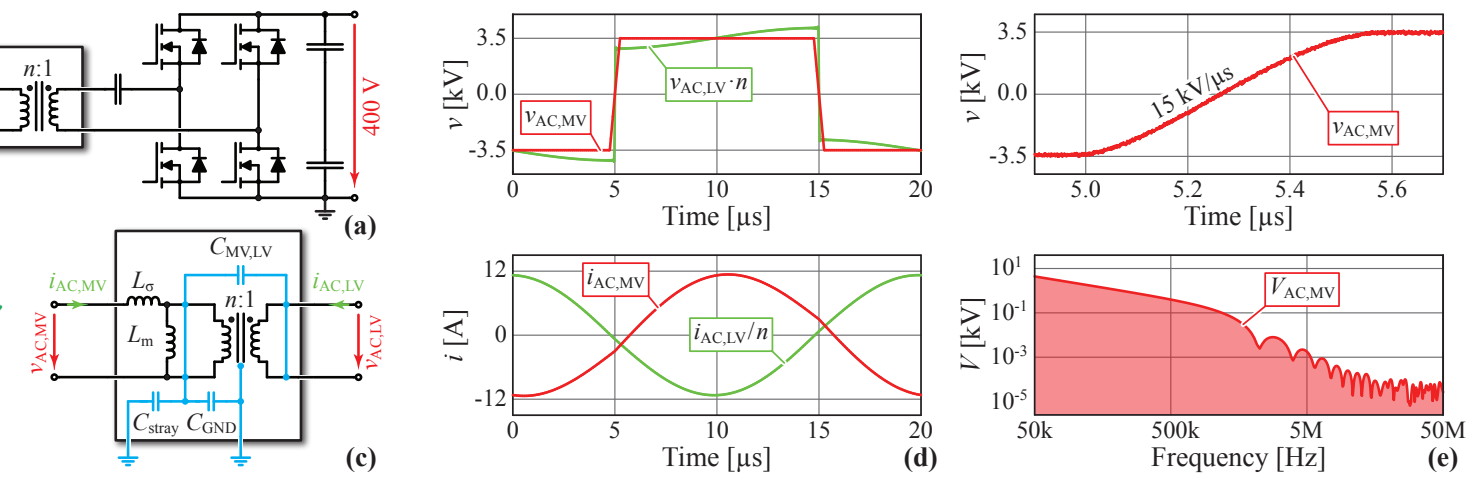

Fig. 1. (a) Series-resonant DC-DC converter, (b) $7 \mathrm{kV}$ MV bridge employing $10 \mathrm{kV} \mathrm{SiC} \mathrm{MOSFETs,} \mathrm{and} \mathrm{(c)} \mathrm{MV/MF} \mathrm{transformer} \mathrm{equivalent} \mathrm{circuit} \mathrm{with} \mathrm{the}$ relevant CM capacitances. (d) Simulated transformer voltages and currents. (e) Measured ZVS transition for the MV side with the corresponding RMS spectrum envelope [20].

TABLE I

PARAMETERS OF THE MV/MF TRANSFORMER.

\begin{tabular}{ll}
\hline Power & $25 \mathrm{~kW} / 31 \mathrm{kVA}$ \\
Voltage & $\pm 3.5 \mathrm{kV} / \pm 400 \mathrm{~V}$ \\
Frequency & $50 \mathrm{kHz}$ \\
Switching & $\mathrm{ZVS} / 15 \mathrm{kV} / \mu \mathrm{s}$ \\
Topology & series-resonant DC-DC converter \\
$L_{\sigma} / L_{\mathrm{m}}$ & $180 \mu \mathrm{H} / 4.5 \mathrm{mH} / \mathrm{MV}$ side \\
\hline Winding & $54: 6 \mathrm{turns} /$ litz wire $/$ shell-type \\
MV wire & $630 \times 71 \mu \mathrm{m} /$ three layers $/$ two chambers \\
LV wire & $2500 \times 100 \mu \mathrm{m} /$ single layer \\
\hline Core type & ferrite BFM8 / U-core $/ 2500 \mathrm{~mm}^{2}$ \\
Core window & $32 \times 61 \mathrm{~mm}$ \\
Air gap & $2 \times 1 \mathrm{~mm}$ \\
\hline Cooling & conduction / forced convection \\
Insulation & silicone elastomere $/ \varepsilon_{\mathrm{r}}=3.0 / 15 \mathrm{kV}$ \\
\hline Volume & $172 \times 122 \times 135 \mathrm{~mm} / 2.8 \mathrm{dm}^{3}$ \\
Losses & $P_{\text {winding }}=40 \mathrm{~W} / P_{\text {core }}=54 \mathrm{~W} / P_{\text {other }}=14 \mathrm{~W}$ \\
Efficiency & $99.55 \% @ 9 \mathrm{~kW} / \mathrm{dm}^{3} /$ simulated \\
\hline
\end{tabular}

load-independent and that the switching transition, shown in Fig. 1(e), features a limited speed [20]. Compared to dual active bridge converters presented in [4], [9], this reduced switching speed limits the CM currents, the EMI emission, and the excitation of transformer resonances. Moreover, this topology does not require a specific leakage inductance for the transformer since the resonance frequency can be adjusted with the resonant capacitor.

Different possibilities exist for the earthing of the MV side: solid-earthing, resistive earthing, or unearthed operation [21]. Furthermore, different connecting nodes can be considered for the earthing: DC-link mid-point, DC-link negative rail, nodes placed outside the DC-DC converter (e.g. DC-DC converter with an AC-DC front-end). In this paper, the DClink negative rail is earthed (solid earthing).

\section{B. MVIMF Transformer}

Fig. 2 depicts the considered MV/MF transformer which is designed according to the guidelines presented in [3], [5] with a target efficiency of $99.5 \%$. The characteristic properties of the transformer are summarized in Tab. I and the main design choices are presented below.

In order to obtain enough current for ZVS, the magnetizing inductance of the transformer should be reduced. For this reason, an air gap is introduced between the core halves (for all limbs). A U-core based transformer allows an efficient cooling of the winding near the winding head. A shell-type winding arrangement reduces the stray field in the winding window and, therefore, the high-frequency winding losses. The LV winding is placed near the wound limb for several reasons: this winding arrangement improves the cooling of the MV winding, which is critical [11], the capacitance between the MV winding and the core is reduced, and the realization of the MV cable terminations is facilitated. The MV winding is divided in two chambers and three layers which represents a good trade-off between the insulation stresses, the winding capacitance, and the complexity of the winding scheme [22]. Two air ducts are placed between the core window and the windings to allow for an efficient cooling [3].

The transformer core is earthed with respect to the LV side (cf. Fig. 1(c)) such that no insulation is required for the $\mathrm{LV}$ winding. Even if the applied DM voltage to the MV winding is $\pm 3.5 \mathrm{kV}$, a CM voltage (DC and AC) is present due to the MV side earthing scheme and the presence of a half-bridge of the MV side (cf. Fig. 1(a)). This means that the total applied voltage to the transformer insulation is $7 \mathrm{kV}$ (the dry-type insulation is rated for $15 \mathrm{kV}$ ).

The relevant $\mathrm{CM}$ capacitances of the transformer are shown in Fig. 1(c) where $C_{\mathrm{MV}, \mathrm{LV}}$ is the capacitance between the windings, $C_{\mathrm{GND}}$ the capacitance between the MV winding and the earth connection of the transformer (core), and $C_{\text {stray }}$ the parasitic capacitance between the MV winding and earthed element placed outside the frame of the transformer. In this paper, a copper plane, which represents the PCB of the LV bridge, is placed on the top of the transformer (cf. Fig. 2). This plane is used for the evaluation of the magnetically and electrically coupled disturbances. 

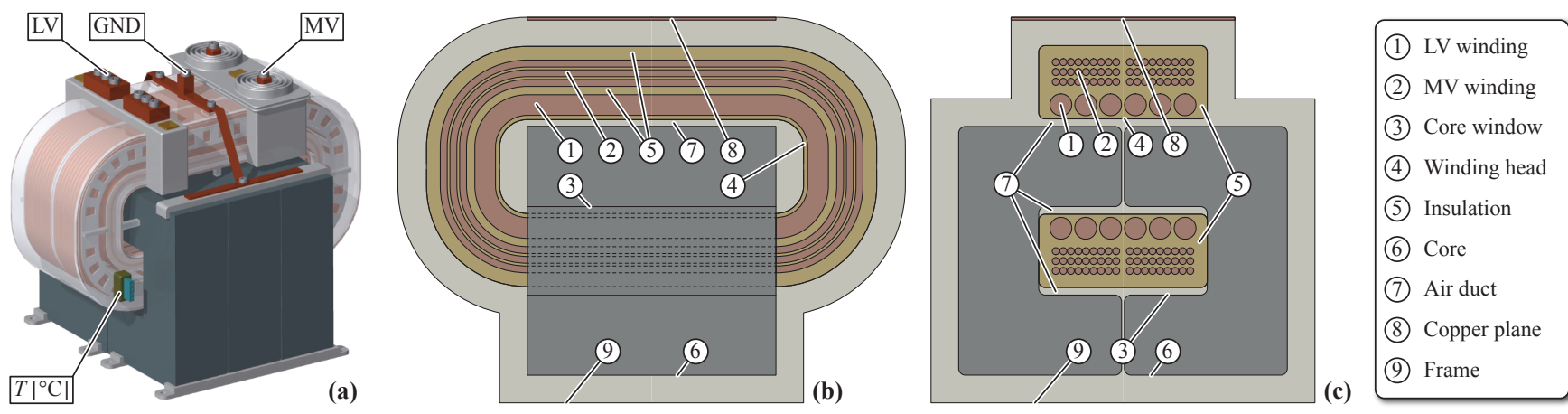

Fig. 2. (a) 3D view of the considered U-core shell-type transformer. (b) Schematic cut side view and (c) cut core window view of the transformer. The (non-conductive) frame is placed $10 \mathrm{~mm}$ away from the transformer boundaries. At the top of the frame, a copper plane $(90 \times 80 \mathrm{~mm}, 75 \mu \mathrm{m})$ is placed. This plane, which represents the PCB of the LV converter, is used to evaluate the coupled disturbances.
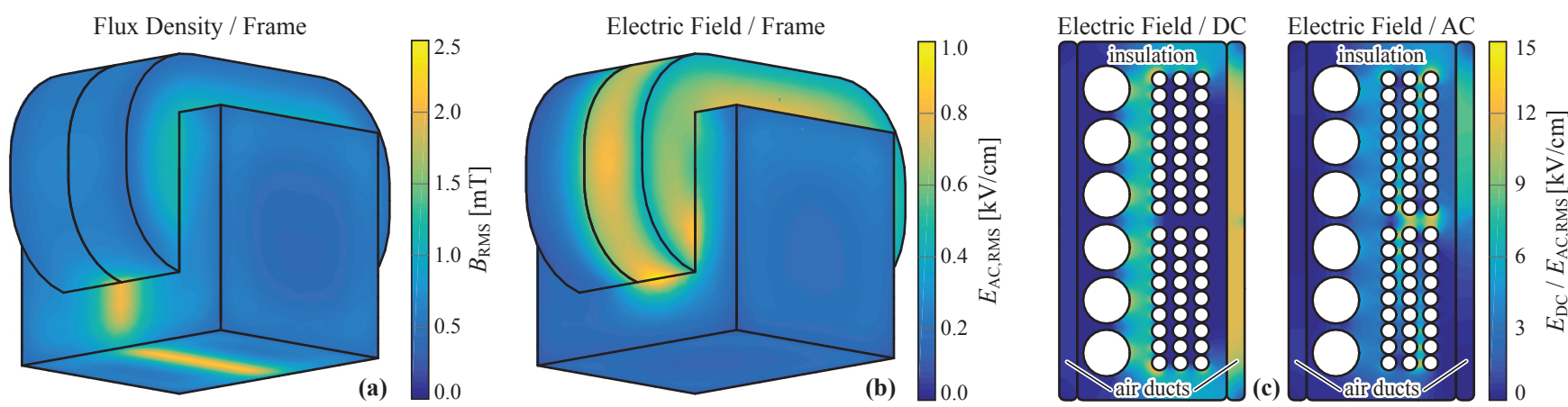

Fig. 3. (a) Magnetic flux density and (b) AC electric field evaluated at the surface of the (non-conductive) frame of the transformer which is placed $10 \mathrm{~mm}$ away from the transformer boundaries. (c) DC and AC electric fields evaluated inside the core window (insulation and air ducts).

TABLE II

Nomenclature (Voltages, CURRENTS, AND $\{E, B\}$ FIELDS).

\begin{tabular}{ll}
\hline$\{V, I\}$ & RMS value \\
$\{v, i\}$ & transient value \\
$\{V, I\}_{\text {peak }}$ & peak transient value \\
$\{\underline{V}, \underline{I}\}$ & complex RMS phasor \\
\hline$\{E, B\}_{\mathrm{RMS}}$ & RMS value (AC harmonics and DC component) \\
$\{E, B\}_{\mathrm{AC}, \mathrm{RMS}}$ & RMS value (AC harmonics) \\
$\{E, B\}_{\mathrm{DC}}$ & DC component \\
\hline$\{E, B\}_{\mathrm{RMS}, \max }$ & local maximum of $\{E, B\}_{\mathrm{RMS}}$ \\
$\{E, B\}_{\mathrm{AC}, \mathrm{RMS}, \max }$ & local maximum of $\{E, B\}_{\mathrm{AC}, \mathrm{RMS}}$ \\
$\{E, B\}_{\mathrm{DC}, \max }$ & local maximum of $\{E, B\}_{\mathrm{DC}}$ \\
$\{E, B\}_{\text {peak,max }}$ & local maximum of the peak transient value \\
\hline
\end{tabular}

\section{Magnetic/EleCtRiC Field ANALYsis}

With these data, it is possible to compute the magnetic and electric fields by means of 3D frequency-dependent FEM analysis using the waveforms of Figs. 1(d) and (e), i.e. including the harmonics. In this Section, the transformer is considered without any shielding. Tab. II denotes the nomenclature used for the description of the voltages, currents, electric fields, and magnetic flux densities. Figs. 3(a) and (b) depict the magnetic flux density and the AC electric field obtained at the surface of the non-conductive frame of the transformer (cf. Fig. 2). Fig. 3(c) shows the DC and AC electric fields in the core window (insulation and air ducts), where the highest electric field values are located (due to the equipotential surface defined by the earthed core).

Due to the large magnetizing current of the transformer (which forms a magnetic dipole), a magnetic stray field exists outside the transformer. As expected, the magnetic flux density is maximal $\left(B_{\text {peak,max }}=3.1 \mathrm{mT}\right)$ near the air gap, where the field is concentrated. This stray magnetic field can cause eddy current losses in surrounding conductive elements and EMI perturbations in electronic circuits [3], [14]. In the considered copper plane (cf. Fig. 2), placed at the top of the transformer, the eddy current losses are $0.9 \mathrm{~W}$. It has to be noted that the considered plane is magnetically thin. For a thicker plane, the losses can exceed 10W. The stray magnetic field can be reduced if the air gap is only placed at the wound limb or with a magnetic shield placed between the transformer and the sensitive circuits [23].

However, the magnetic flux density is not a problem which is specific to MV transformers and has already been examined [14], [15]. Therefore, the magnetic shielding is not further examined. In contrast, the electric field, which is directly related to the applied voltage, creates several issues.

- DC field: The DC electric field distribution is determined by the conductivity and not by the permittivity [17], [18]. The surface conductivity of the insulation is not clearly defined and the conductivity of air depends on many environmental factors [24]. Therefore, the DC field distribution is ill-defined. Since the bulk conductivity of 

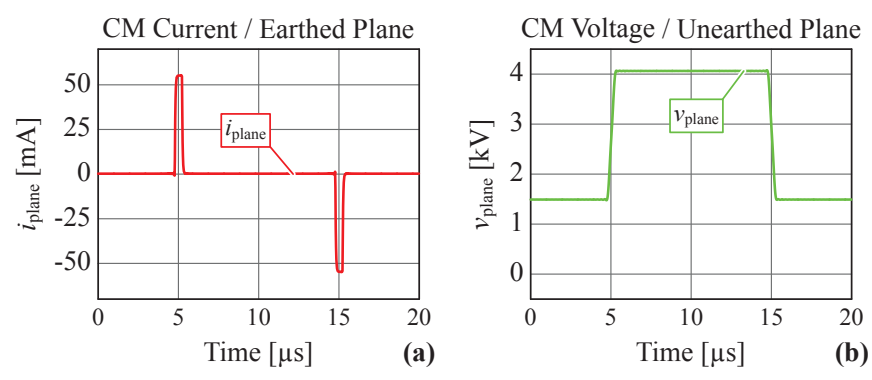

Fig. 4. (a) Capacitively coupled CM current in the earthed copper plane (b) Capacitively coupled CM voltage in the unearthed copper plane. The plane is placed near the winding head (cf. Fig. 2).

the insulation is usually larger than the conductivity of air, a large part of the DC electric field can be located in the air. This is particularly critical in the air ducts, due to the small distances (cf. Fig. 3(c)).

- AC field: If the frequency is higher than some $\mathrm{mHz}$, the electric field distribution is determined by the permittivity [25]. Still, due to the permittivity mismatch between the insulation material and the air, a significant part of the AC electric field is located in the air Again, this field displacement is large in the air ducts (cf. Fig. 3(c)).

- Surface field: The total electric field (DC and AC) in the air is maximal at the surface of the air ducts (cf. Fig. 3(c)) and reaches a value of $E_{\text {peak,max }}=21.2 \mathrm{kV} / \mathrm{cm}$. This value, which is close to the critical field in air [17], can lead to surface discharges (creeping with tangential field). Such discharges damage the insulation, distort the potential distribution, and can lead to a flashover [19], [26], [27]. The triple points, i.e. the locations at the interfaces between the insulation, the core, and the air, are particularly sensitive [19], [28], [29]. Moreover, the MF and HF (due to the switching transients) harmonics applied to the insulation further reduce the lifetime of the transformer in the presence of discharges [10], [28], [29]. In reality, the electric field values are usually larger than the simulated values due to imperfections of the potting process, surface roughness, unexpected triple points, etc. Furthermore, the values presented in Fig. 3 can be exceeded during abnormal operating situations, e.g. when a voltage drop is present between the $\mathrm{LV}$ and the MV earthing of the converter [21].

- Near-field: The AC electric field, evaluated at the surface of the frame, is mainly located near the winding head and reaches a value of $E_{\mathrm{AC}, \mathrm{RMS}, \max }=1.0 \mathrm{kV} / \mathrm{cm}$ (cf. Fig. 3(b)). If grounded conductors are placed near the frame, the electric field would be even higher. Since this field contains HF components (cf. Fig. 1(e)), this can create perturbations in electronic circuits or in the cooling system of the transformer [14].

Figs. 4(a) and (b) show the capacitively coupled current and voltage in the considered copper plane placed near the transformer (cf. Fig. 2). If the plane is earthed, the displacement current $\left(I_{\text {plane,peak }}=59.2 \mathrm{~mA}\right)$ can cause substantial perturbations, especially for sensors. For an unearthed plane, the coupled voltage $\left(V_{\text {plane,peak }}=4.1 \mathrm{kV}\right)$ produces an insulation coordination issue.

The simplest solution for reducing the surface electric field is to increase the thickness of the insulation. The capacitively coupled disturbances are reduced if the distances between the transformer and the sensitive circuits is increased. However, these solutions reduce the achievable power density and causes thermal issues. For these reasons, another option, which is the shielding of the transformer [12], [30], is examined in detail in the following.

\section{Electrical SHIELDiNG}

\section{A. Shielding Methods}

Field control (grading) is well-established for lowfrequency MV components (e.g. bushings or machine stators) [28], [31]. Different methods can be used to shape the electric field distribution and can be classified as follows [17], [19], [32].

- Geometric: A conductive electrode with a large curvature radius is placed in order to shape the electric field with a defined equipotential surface.

- Capacitive: Many conductive electrodes are placed (often in a concentric configuration) in order to define many equipotential surfaces. The voltage distribution between the electrodes is defined by the capacitance matrix.

- Resistive: A semi-resistive (semi-conducting) material is used in order to change the impedance of the insulation system and, therefore, the potential distribution. Often non-linear resistive materials are used (conductivity is electric field dependent).

- Refractive: A material with a high permittivity is placed at the surface of the insulation in order to shape the electric field (field displacement).

All these methods are applicable for the field grading of low-frequency AC components. In [33], [34], it has been shown that low-frequency field grading systems can produce dramatic failures in the presence of MF or DC electric fields. Therefore, for the shielding of MV/MF transformers, additional constraints appear as follows.

- Compactness: Due to the reduced dimension of the transformer (compared to standard low-frequency components), complex field grading methods are difficult to implement (e.g. methods based on multiple conductive layers).

- Frequency: The shield should be active at DC/MF/HF. The grading methods relying on the permittivity (geometric, capacitive, and refractive) are not easily applicable for DC fields. Resistive gradings (particularly with non-linear materials) are difficult to design for a wide frequency range [28], [34].

- Losses: In [3], it has been shown that MF magnetic fields (cf. Fig. 3(a)) produce significant losses in metallic elements used for heat management. This is critical for the grading methods using metallic electrodes (geometric, 

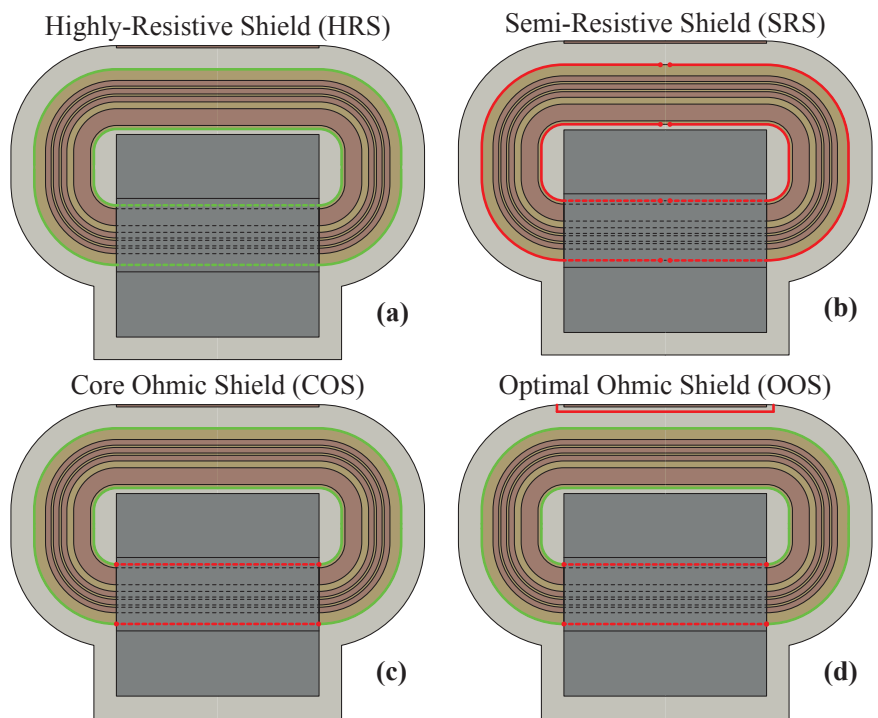

Highly-resistive coating Semi-resistive coating

Non-conducting surface

Fig. 5. Different resistive shielding methods for the transformer (cf. Fig. 2(b)). (a) Highly-Resistive Shield (HRS), (b) Semi-Resistive Shield (SRS), (c) Core Ohmic Shield (COS), and (d) Optimal Ohmic Shield (OOS).

capacitive) [30]. Furthermore, the displacement current flowing in a resistive material used for field grading can also produce losses at MF [28], [33].

\section{B. Resistive Shielding}

Taking all the aforementioned factors into account, it has been decided to place a (linear) resistive shield around both windings. Different configurations are possible for the shielding.

- No Shielding (NS): The transformer is considered without any shielding, as shown in Fig. 2(b).

- Highly-Resistive Shield (HRS): The surface of the winding insulation (around both windings) is covered with a highly-resistive coating, as shown in Fig. 5(a).

- Semi-Resistive Shield (SRS): The surface of the winding insulation (around both windings) is covered with a semi-resistive coating, as shown in Fig. 5(b). Two small gaps are inserted to prevent the formation of a closed current path for eddy currents (short-circuit winding).

- Core Ohmic Shield (COS): The surface of the winding insulation (around both windings) near the core window, is covered with a semi-resistive coating. Moreover, a highly-resistive coating is used near the winding head, as shown in Fig. 5(c).

- Optimal Ohmic Shield (OOS): Similar with respect to the COS. An additional semi-resistive shield is used near the copper plane (cf. Fig. 2), as shown in Fig. 5(d).

All the resistive shields are connected to the core (and therefore to the earth) and are easily obtained by a surface treatment of the insulation surface. It has to be noted that the shields should be terminated with a finite curvature radius in order to avoid an electric field hotspot (triple point) [19], [32]. Terminations of the shield are required at the following locations: cable terminations (bushings),

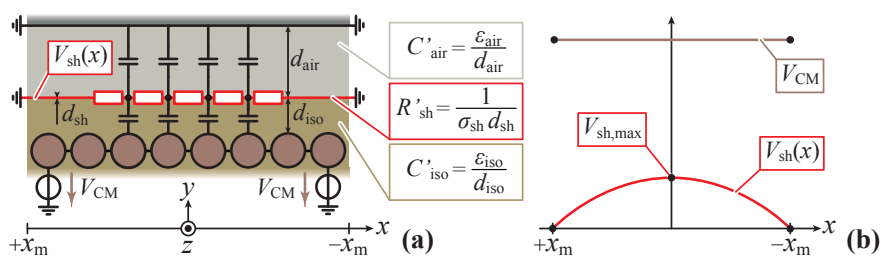

Fig. 6. (a) Electric equivalent circuit of an earthed resistive shield excited by a CM voltage source. (b) Voltage drop along the shield for the case $V_{\text {sh,max }} \ll V_{\mathrm{CM}}$.

shield gaps (SRS), transition between a semi-resistive and a highly-resistive shield (COS and OOS), etc.

\section{Shield Functional Principle}

The computation of the shielding effect and the losses of the inserted resistive elements is required for the evaluation of the proposed shielding methods. The shield is primarily defined by the surface conductivity of the coating [35]:

$$
\sigma_{\mathrm{s}, \mathrm{sh}}=\sigma_{\mathrm{sh}} d_{\mathrm{sh}}
$$

where $\sigma_{\mathrm{sh}}$ is the bulk conductivity of the resistive material and $d_{\text {sh }}$ the thickness of the coating.

If the shield forms a short-circuit winding (e.g. HRS), the shield can be seen as a third winding with one turn. The load of this third winding is the resistance of the complete shield. In order to have low losses, the resistance of the shield should be very high compared to the load impedance of the transformer (equivalent impedance referred to the short-circuit winding with one turn). Even if no short-circuit winding is present (e.g. HRS, COS, and OOS), the stray magnetic field of the transformer will still induce eddy current losses (cf. Fig. 3(a)). In order to limit the losses in the shield, the skin depth should be much larger than the thickness of the shield:

$$
\delta_{\mathrm{sh}}=\sqrt{\frac{2}{2 \pi f \mu_{0} \sigma_{\mathrm{sh}}}} \gg d_{\mathrm{sh}},
$$

where $f$ is the switching frequency. It has to be noted that this condition is required but not sufficient to have low losses. Even a magnetically thin layer can produce eddy current losses as calculated in [36] for a simple geometry. Since the shield of the transformer features a complex geometry and field distribution (tangential and orthogonal), numerical simulations are required for computing the eddy current losses.

Fig. 6 shows the electrical equivalent circuit of the shield inside the core window (cf. Fig. 2(c)). In the $y$ direction, the MV winding, the insulation, the shield, the air duct, and the core are placed. The shield is earthed at both ends (to the core) and the MV winding potential is changing (CM voltage). The equivalent circuit can be formulated with the following differential equations, which are similar to the telegrapher's equations:

$$
\frac{\partial \underline{V}_{\mathrm{sh}}(x)}{\partial x}=-R_{\mathrm{sh}}^{\prime} \underline{I}_{\mathrm{sh}}(x),
$$




$$
\frac{\partial \underline{I}_{\mathrm{sh}}(x)}{\partial x}=-\mathrm{j} 2 \pi f\left(C_{\mathrm{iso}}^{\prime}\left(\underline{V}_{\mathrm{sh}}(x)-\underline{V}_{\mathrm{CM}}\right)+C_{\mathrm{air}}^{\prime} \underline{V}_{\mathrm{sh}}(x)\right),
$$

where $\underline{V}_{\text {sh }}$ is the voltage along the shield, $\underline{I}_{\mathrm{sh}}$ the current along the shield, $R_{\mathrm{sh}}^{\prime}$ the resistance of the shield, $C_{\mathrm{iso}}^{\prime}$ the insulation capacitance, $C_{\text {air }}^{\prime}$ the air duct capacitance, $\underline{V}_{\mathrm{CM}}$ the applied winding voltage, and $f$ the considered frequency. The different variables are scaled with respect to the depth of the shield in the out-of-place direction ( $z$ direction). The earthing of the shield leads to the boundary conditions:

$$
\underline{V}_{\mathrm{sh}}\left(+x_{\mathrm{m}}\right)=\underline{V}_{\mathrm{sh}}\left(-x_{\mathrm{m}}\right)=0,
$$

where $2 x_{\mathrm{m}}$ is the length of the shield. The solution of this boundary value problem is a large expression but a simple approximation exists (obtained with the perturbation theory) if the voltage drop along the shield is small. This last condition $\left(V_{\mathrm{sh}, \max } \ll V_{\mathrm{CM}}\right)$ has to be respected for obtaining a shielding effect and leads to:

$$
\underline{V}_{\mathrm{sh}}(x) \approx \underbrace{\mathrm{j} 2 \pi f R_{\mathrm{sh}}^{\prime} C_{\mathrm{iso}}^{\prime} \frac{x_{\mathrm{m}}^{2}}{2} \underline{V}_{\mathrm{CM}}}_{\underline{V}_{\mathrm{sh}, \max }}\left(1-\left(\frac{x}{x_{\mathrm{m}}}\right)^{2}\right) .
$$

From this equation, it can be seen that, for a given configuration, the shielding is only efficient up to a maximum frequency. For obtaining, a shielding at high frequencies, a high surface conductivity is required. The losses per unit depth ( $z$ direction) of the shield can be expressed as:

$$
P^{\prime}=\int_{-x_{\mathrm{m}}}^{+x_{\mathrm{m}}} R_{\mathrm{sh}}^{\prime}\left|\underline{I}_{\mathrm{sh}}(x)\right|^{2} \mathrm{~d} x=\int_{-x_{\mathrm{m}}}^{+x_{\mathrm{m}}} \frac{1}{R_{\mathrm{sh}}^{\prime}}\left|\frac{\partial \underline{V}_{\mathrm{sh}}(x)}{\partial x}\right|^{2} \mathrm{~d} x .
$$

This last equation shows that the losses converges to zero with for very high surface conductivities. For very low surface conductivities, the losses are also converging towards zero (no current is flowing in the shield). The losses are maximal when the shield conductivity is not high enough for defining an equipotential surface but large enough for producing losses. Alternatively, the shield can be considered as a resistor-capacitor circuit. The losses are low if the time constant is well below the switching frequency or well above the switching speed. Between these two cases, a non-negligible fraction of energy stored in the insulation capacitance is dissipated in the shield.

Finally, it can be seen (cf. (4)) that only the surface conductivity has an impact on the shielding effect. This offers a degree of freedom (bulk conductivity and thickness) for realizing the optimal surface conductivity such that low losses and an effective shielding are obtained. Nevertheless, the magnetic losses are not scaling directly with thickness of the shield (cf. (1)), such that the magnetic simulations should be conducted with the correct coating thickness.

\section{Optimal Shield Conductivity}

It is concluded that the conductivity of the shield is a trade-off between the losses and the effectiveness of the shielding at HF. In order to find the optimal conductivity, 3D FEM simulations (cf. Fig. 3) are conducted. Fig. 7(a) depicts the losses for the HRS and SRS concepts, which are the two critical configurations with respect to the losses, due to the proximity between the shield and the windings. The simulation are conducted for a shield thickness of $250 \mu \mathrm{m}$. However, the results are very similar for $100 \mu \mathrm{m}$ or $500 \mu \mathrm{m}$.

It is interesting to note that a large part of the eddy current losses in the shield is produced by the magnetizing current, which is much smaller than the load current (cf. Fig. 1(d). This is explained by the fact that the magnetizing current is mainly responsible for the stray field located outside the windings. This also implies that a shield placed between the MV and LV windings, where the stray field is maximal, would lead to high losses. As expected, an electric loss peak is present between very low and very high surface conductivities (cf. (7)). Therefore, the surface conductivities are selected for the different types of shields as follows.

- HRS: Due to the presence of the short-circuit winding, a very low conductivity of $10^{-9} \mathrm{~S}$ is selected (below the electric loss peak). The HRS can be obtained with a typical anti-static surface treatment of the windings (against electrostatic discharges).

- SRS: Due to the limited eddy current losses, a higher conductivity (above the electric loss peak) can be selected. A surface conductivity of $0.05 \mathrm{~S}$ represents a good trade-off between the electric and magnetic losses and can be obtained with a carbon coating.

With the selected conductivities, the losses are less than 1.0W for both shielding types. A closer look at Fig. 7(a) reveals that large tolerances are acceptable for the surface conductivity, which facilitates the coating process.

Fig. 7(b) shows the magnetic flux density and indicates that the shield is magnetically transparent (cf. (2)). Figs. 7 (c) and (d) depict the electric field at the frame and at the surface of the insulation. For very low surface conductivity (e.g. HRS), the total (AC and DC) electric fields are equal to the AC fields shown Figs. 3(b) and (c). This implies that the HRS blocks the DC electric field while the AC field is unaffected. This corresponds to the shielding method presented in [12]. For the SRS, all the relevant frequency components are blocked (DC/MF/HF) and, therefore, this shielding method is further examined.

\section{E. Shielding Effects}

Even if the aforementioned results show that the SRS provides a very effective shielding, some frequency dependence of the potential distribution is still expected (cf. (6)). Fig. 8(a) depicts the frequency dependence of the maximum surface electric field of the transformer for a normalized $\mathrm{CM}$ excitation of the MV winding. The SRS is only effective for frequencies below $10 \mathrm{MHz}$ which is compatible with the spectrum shown in Fig. 1(e). For higher frequencies, a large voltage drop appears along the shield, leading to a non-negligible field in the air ducts (cf. (6)). For the NS/HRS, the field is dropping near $100 \mathrm{MHz}$ due to the limited conductivity of the ferrite core [22]. It has to be noted that, due to resonances of the transformer, at HF, the voltage distribution of the winding cannot be described with a single 

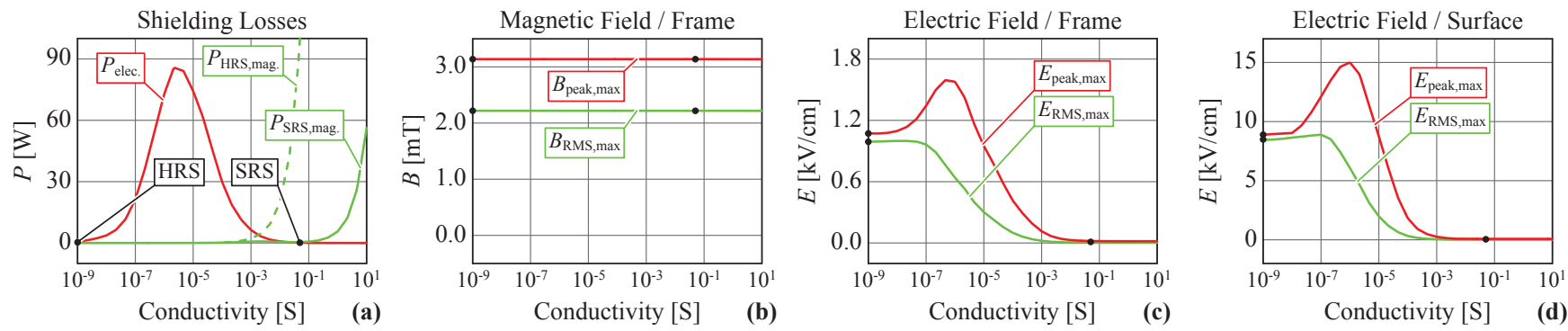

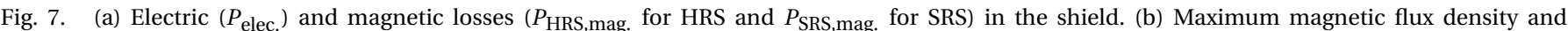
(c) electric field evaluated at the surface of the non-conductive frame of the transformer. (d) Maximum electric field at the surface of the insulation. Two optima are identified: one for the HRS and one for the SRS.
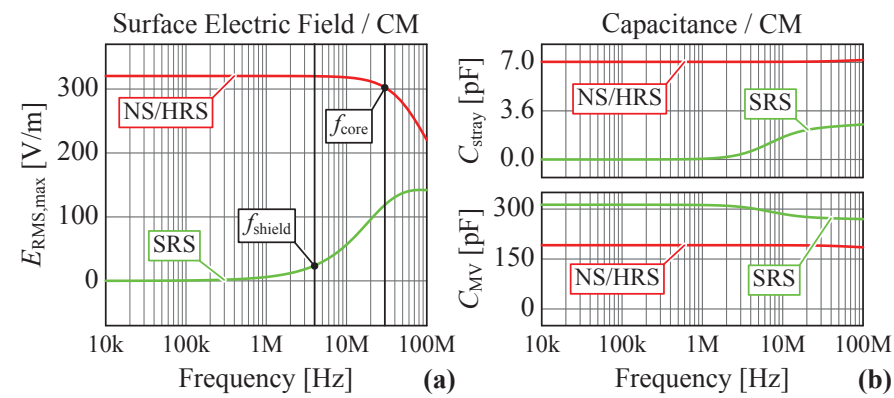

Fig. 8. (a) Maximum surface electric field in the air ducts for a normalized RMS CM excitation of the MV winding $(1 \mathrm{~V})$. Above $f_{\text {core }}$ and $f_{\text {shield }}$, the core and the shield cannot be considered as equipotential surfaces anymore. (b) CM capacitances of the transformer. $C_{\text {stray }}$ is the capacitance between the MV winding and the copper plane. $C_{\mathrm{MV}}$ is the total MV CM capacitance of the transformer which consists of $C_{\mathrm{MV}, \mathrm{LV}}$ and $C_{\mathrm{GND}}$ (cf. Fig. 1(c)).

a CM/DM voltage [4], [34]. However, the aforementioned $\mathrm{CM}$ excitation is still useful to assess the shielding effect without considering the transformer resonances.

Fig. 8(b) shows the CM capacitance between the MV winding and the earthed copper plane and the total MV CM capacitance, which consists of the CM capacitance towards the LV winding and the core (cf. Fig. 1(c)). For both curves, the effect of the limited shield conductivity also affects the capacitances at HF. The SRS drastically reduces the stray capacitance towards the copper plane, suppressing potential disturbance couplings (cf. Fig. 4). However, the SRS increases the total CM capacitance of the transformer by $60 \%$. This increased capacitance results from the field confinement provided by the shield (which is connected to the core), particularly near the winding head (cf. Fig. 3(b) and Fig. 6(a)). An increase of the CM capacitance is often problematic since it can create conducted EMI perturbations inside the converter. This can be handled with $\mathrm{CM}$ chokes placed at the transformer's AC terminals or at the DC-buses [8].

An alternative solution is to use the COS concept (cf. Fig. 5(c)) in order to mitigate the increase of the total MV CM capacitance. This concept uses a semi-resistive shield inside the core window in order to reduce the surface electric field (surface conductivity of $0.05 \mathrm{~S}$, similar to SRS). This shield does not significantly increase the total MV CM capacitance since the core already defines the potential in this region. Near the winding head, where the surface electric field is lower, a highly-resistive anti-static shield is used for blocking the DC field (surface conductivity of $10^{-9} \mathrm{~S}$, similar to HRS). With the COS, the total MV CM capacitance only increases by $10 \%$. However, this concept is not able to limit the parasitic capacitance towards the copper plane (cf. Fig. 4).

In order to limit the capacitively coupled disturbances, the OOS concept (cf. Fig. 5(d)), which features an additional semi-resistive shield near the copper plane, can be used (surface conductivity of $0.05 \mathrm{~S}$, similar to SRS). Since this shield is placed near the frame and not near the transformer windings, the total MV CM capacitance is only marginally affected. Alternatively, the complete transformer frame could be coated with a resistive coating for blocking the electric field without producing eddy current losses [32].

Tab. III summarizes the properties of the different shielding methods with respect to the magnetic flux density, electric field, capacitances, etc. The HRS blocks the DC field and, therefore, reduces the surface electric field. However, the AC field, which causes the capacitively coupled disturbances, remains unaffected. The SRS drastically limits the surface electric field and the capacitively coupled voltages or currents. Nevertheless, the coupling capacitance of the transformer is increased. The COS reduces the surface electric field without increasing the transformer coupling capacitance but does not prevent capacitively coupled disturbances. Finally the OOS significantly decreases the surface electric field and the capacitively coupled disturbances without increasing the transformer coupling capacitance.

\section{F. Experimental Verification}

The SRS is validated with the transformer presented in [4], which features similar ratings $( \pm 4 \mathrm{kV} / \pm 400 \mathrm{~V}, 50 \mathrm{kHz}, 25 \mathrm{~kW})$, cf. Fig. 9(a). The transformer has first been measured without shielding (NS). In a second step, a carbon based coating (four coats, connected to the core) is added for obtaining a shield (SRS) with a measured surface conductivity of $220 \Omega$ [37]. No additional losses are measured with the SRS, as long as the shield does not form a short-circuit winding.

The equivalent circuit of the transformer and the measurement setup are shown in Fig. 9(b) where a closed metallic frame (copper) is placed $7 \mathrm{~mm}$ away from the transformer boundaries in order to quantity the near electric field. The 


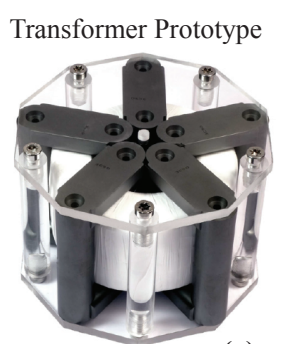

(a)

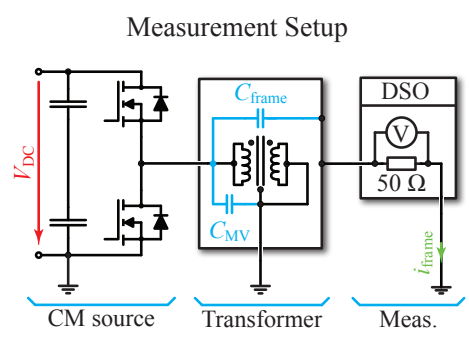

(b)

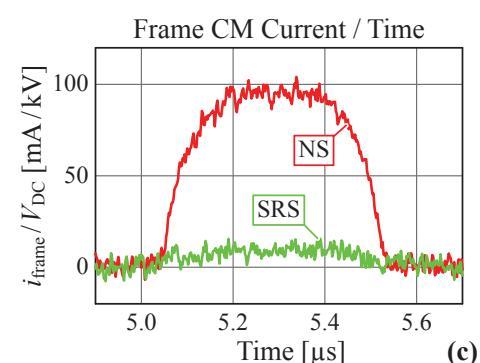

(c)

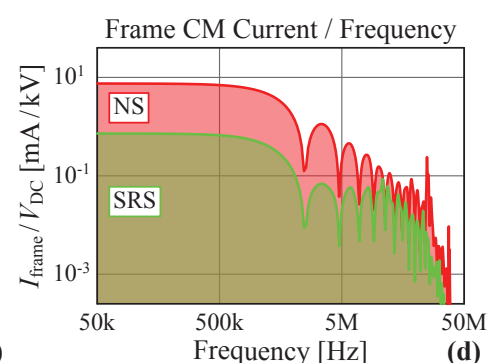

(d)

Fig. 9. (a) Considered transformer prototype [4]. (b) Measurement setup for the CM current in the metallic frame. (c) Measured displacement current through the frame and (d) RMS spectrum envelope of the measured current.

TABLE III

COMPARISON OF THE SHIELDING METHODS.

\begin{tabular}{|c|c|c|c|c|c|}
\hline Type & NS $^{1}$ & HRS $^{2}$ & SRS $^{3}$ & $\cos ^{4}$ & OOS $^{5}$ \\
\hline \multicolumn{6}{|c|}{ Losses / shield / cf. Fig. 7(a) } \\
\hline$P[\mathrm{~W}]$ & 0.0 & 0.1 & 0.7 & 0.5 & 0.5 \\
\hline \multicolumn{6}{|c|}{ Magnetic flux density / frame / cf. Fig. 3(a) } \\
\hline$B_{\mathrm{RMS}, \max }[\mathrm{mT}]$ & 2.2 & 2.2 & 2.2 & 2.2 & 2.2 \\
\hline$B_{\text {peak,max }}[\mathrm{mT}]$ & 3.1 & 3.1 & 3.1 & 3.1 & 3.1 \\
\hline \multicolumn{6}{|c|}{ Electric field / frame / cf. Fig. 3(b) } \\
\hline$E_{\mathrm{DC}, \max }[\mathrm{kV} / \mathrm{cm}]$ & 1.4 & $<0.1$ & $<0.1$ & $<0.1$ & $<0.1$ \\
\hline$E_{\mathrm{RMS}, \max }[\mathrm{kV} / \mathrm{cm}]$ & 1.7 & 1.0 & $<0.1$ & 0.9 & 0.9 \\
\hline$E_{\text {peak,max }}[\mathrm{kV} / \mathrm{cm}]$ & 2.4 & 1.1 & $<0.1$ & 1.0 & 1.0 \\
\hline \multicolumn{6}{|c|}{ Electric field / surface / cf. Fig. 3(c) } \\
\hline$E_{\mathrm{DC}, \max }[\mathrm{kV} / \mathrm{cm}]$ & 14.6 & $<0.1$ & $<0.1$ & $<0.1$ & $<0.1$ \\
\hline$E_{\mathrm{RMS}, \max }[\mathrm{kV} / \mathrm{cm}]$ & 16.0 & 8.5 & $<0.1$ & 4.2 & 4.2 \\
\hline$E_{\text {peak,max }}[\mathrm{kV} / \mathrm{cm}]$ & 21.2 & 8.7 & $<0.1$ & 4.3 & 4.3 \\
\hline \multicolumn{6}{|c|}{ Coupling / copper plane / cf. Figs. 4(a) and (b) } \\
\hline$I_{\text {plane }}[\mathrm{mA}]$ & 11.1 & 11.1 & $<0.1$ & 10.9 & $<0.1$ \\
\hline$I_{\text {plane,peak }}[\mathrm{mA}]$ & 59.5 & 59.5 & $<1.0$ & 59.2 & $<1.0$ \\
\hline$V_{\text {plane }}[\mathrm{kV}]$ & 3.1 & 3.1 & $<0.1$ & 3.0 & $<0.1$ \\
\hline$V_{\text {plane,peak }}[\mathrm{kV}]$ & 4.1 & 4.1 & $<0.1$ & 4.0 & $<0.1$ \\
\hline \multicolumn{6}{|c|}{ Capacitance / CM / cf. Fig. 8(b) } \\
\hline$C_{\text {stray }}[\mathrm{pF}]$ & 7 & 7 & $<0.1$ & 7 & $<0.1$ \\
\hline$C_{\mathrm{MV}}[\mathrm{pF}]$ & 191 & 191 & 313 & 208 & 214 \\
\hline \multicolumn{6}{|c|}{${ }^{1}$ NS: No Shielding $(0.0 \mathrm{~S})$} \\
\hline \multicolumn{6}{|c|}{${ }^{2}$ HRS: Highly-Resistive Shield $\left(10^{-9} \mathrm{~S}\right)}$. \\
\hline \multicolumn{6}{|c|}{${ }^{3}$ SRS: Semi-Resistive Shield (0.05S). } \\
\hline \multicolumn{6}{|c|}{${ }^{4}$ COS: Core Ohmic Shield $\left(10^{-9} \mathrm{~S} / 0.05 \mathrm{~S}\right)$} \\
\hline \multicolumn{6}{|c|}{${ }^{5}$ oOS: Optimal Ohmic Shield $\left(10^{-9} \mathrm{~S} / 0.05 \mathrm{~S}\right)$} \\
\hline
\end{tabular}

energy stored in the near-field (between the transformer and the frame) is related to the capacitance towards the frame. First, the total CM capacitance of the transformer $\left(C_{\mathrm{MV}}\right)$ has been measured. As expected the SRS increases the total CM capacitance (from $151 \mathrm{pF}$ to $228 \mathrm{pF}$ ). For assessing the shielding effect, the parasitic capacitance towards the frame $\left(C_{\text {frame }}\right)$ is measured. With the shield, the parasitic capacitance is significantly reduced (from $38 \mathrm{pF}$ to $4 \mathrm{pF}$ ), indicating that the electric field is confined in the insulation.

The displacement current has also been measured in the time domain. The MV winding is excited with a rectangular $\mathrm{CM}$ voltage with $450 \mathrm{~ns}$ rise and fall times (cf. Fig. 1(e)) and an amplitude of $200 \mathrm{~V}$. The CM current in the frame is measured through a $50 \Omega$ resistor (cf. Fig. 9(b)), given that the impedance of the frame capacitance is much larger than the $50 \Omega$ resistor (for frequencies below $50 \mathrm{MHz}$ ). Figs. 9(c) and (d) show that the measured displacement current and the spectrum envelope, respectively. It can be concluded that the shield is effective in the frequency range where significant voltage harmonics exist.

\section{Conclusion}

The increased voltage, frequency, and switching speed achieved by modern SiC devices are generating critical voltage transients inside MV/MF transformers. In this paper, a $\pm 3.5 \mathrm{kV} / \pm 400 \mathrm{~V}, 50 \mathrm{kHz}, 25 \mathrm{~kW}$ transformer used in a DCDC converter has been analyzed with respect to the shielding of electric fields. It is found that capacitively coupled voltages (peak value of $4.1 \mathrm{kV}$ ) and currents (peak value of $59.5 \mathrm{~mA}$ ) can cause severe disturbances for circuitry placed near the transformer. Additionally, the electric field at the surface of the insulation is very high (peak value of $21.2 \mathrm{kV} / \mathrm{cm}$ ) and can lead to surface discharges which damage the insulation.

The main challenge for the shielding of MV/MF transformers is to find a concept which is able to provide a shielding at $\mathrm{DC} / \mathrm{MF} / \mathrm{HF}$ without adding significant eddy current losses. This implies that metallic materials cannot be used near the transformer. It is shown that a resistive shielding with the appropriate conductivity confines the electric field in the insulation without producing additional ohmic or eddy current losses. Furthermore, an optimal placement of the shield does not increase the coupling capacitance of the transformer in a significant way. This shielding allows the usage of the transformer in EMI sensitive environments and provides a better electric field distribution from an insulation coordination point of view. Furthermore, this shielding concept can be used for other devices, such as power modules, inductors, cooling systems, etc.

However, the shield, which prevents capacitively coupled disturbances, does not, suppress the coupling capacitance of the transformer itself. This means that conducted EMI is still present in the power section of the converter. For this reason, future research will investigate the complete converter, including CM chokes, transformer resonances, loop inductances, earthing resistors, etc. This should be considered for assessing the impact of the capacitive CM/DM currents, originating or flowing through the transformer, on the converter operation. 


\section{ACKNOWLEDGMENT}

This work is part of the Swiss Competence Centers for Energy Research (SCCER) initiative which is supported by the Swiss Commission for Technology and Innovation (CTI).

\section{REFERENCES}

[1] A. K. Tripathi, K. Mainali, D. C. Patel, A. Kadavelugu et al., "Design Considerations of a 15-kV SiC IGBT-Based Medium-Voltage HighFrequency Isolated DC-DC Converter," IEEE Trans. Ind. Appl., vol. 51, no. 4, pp. 3284-3294, Jul. 2015.

[2] D. Rothmund, G. Ortiz, and J. W. Kolar, "SiC-based Unidirectional Solid-State Transformer Concepts for Directly Interfacing 400V DC to Medium-Voltage AC Distribution Systems," in Proc. of the IEEE Telecommunications Energy Conf. (INTELEC), Sep. 2014.

[3] G. Ortiz, M. Leibl, J. W. Kolar, and O. Apeldoorn, "Medium Frequency Transformers for Solid-State-Transformer Applications - Design and Experimental Verification," in Proc. of the IEEE Conf. Power Electronics and Drive Systems (PEDS), Apr. 2013, pp. 1285-1290.

[4] D. Rothmund, G. Ortiz, T. Guillod, and J. W. Kolar, "10kV SiC-Based Isolated DC-DC Converter for Medium Voltage-Connected Solid-State Transformers," in Proc. of the IEEE Applied Power Electronics Conf and Expo. (APEC), Mar. 2015, pp. 1096-1103.

[5] F. Kieferndorf, U. Drofenik, F. Agostini, and F. Canales, "Modular PET, Two-Phase Air-Cooled Converter Cell Design and Performance Evaluation with 1.7kV IGBTs for MV Applications," in Proc. of the IEEE Applied Power Electronics Conf. and Expo. (APEC), Mar. 2016, pp $472-479$.

[6] L. Heinemann, “An Actively Cooled High Power, High Frequency Transformer with High Insulation Capability," in Proc. of the IEEE Applied Power Electronics Conf. and Expo. (APEC), Mar. 2002, pp 352-357.

[7] S. B. Y. Du, G. Wang, and S. Bhattacharya, "Design Considerations of High Voltage and High Frequency Transformer for Solid State Transformer Application," in Proc. of the Industrial Electronics Society Conf. (IECON), Nov. 2010, pp. 421-426.

[8] J. E. Huber and J. W. Kolar, "Common-Mode Currents in MultiCell Solid-State Transformers," in Proc. of the International Power Electronics Conf. (IPEC), May 2014.

[9] S. Madhusoodhanan, A. Tripathi, D. Patel, K. Mainali et al., "Solid State Transformer and MV Grid Tie Applications Enabled by $15 \mathrm{kV}$ SiC IGBTs and $10 \mathrm{kV}$ SiC MOSFETs Based Multilevel Converters," IEEE Trans. Ind. Appl., vol. 51, no. 4, pp. 3343-3360, Jul. 2015.

[10] P. Wang, A. Cavallini, and G. Montanari, "The Influence of Repetitive Square Wave Voltage Parameters on Enameled Wire Endurance," IEEE Trans. Dielectr. Electr. Insul., vol. 21, no. 3, pp. 1276-1284, Jun. 2014

[11] T. Guillod, R. Färber, F. Krismer, C. M. Franck, and J. W. Kolar "Computation and Analysis of Dielectric Losses in MV Power Electronic Converter Insulation," in Proc. of the IEEE Energy Conversion Congr and Expo. (ECCE), Sep. 2016.

[12] T. Guillod, J. E. Huber, G. Ortiz, A. De et al., "Characterization of the Voltage and Electric Field Stresses in Multi-Cell Solid-State Transformers," in Proc. of the IEEE Energy Conversion Congr. and Expo. (ECCE), Sep. 2014, pp. 4726-4734.

[13] N. Soltau, M. Kaymak, S. Cui, and R. W. De Doncker, "Materials, Devices and Components for Flexible Electrical Networks of the Future," in Proc. of the International ETG Congress, Nov. 2015, pp. 1-8.

[14] C. R. Paul, Introduction to Electromagnetic Compatibility. Wiley, 2006

[15] P. Wallmeier and H. Grotstollen, "Magnetic Shielding Applied to High-Frequency Inductors," in Proc. of the IEEE Industry Applications Conference (IAS), vol. 2, Oct. 1997, pp. 1131-1138.

[16] C. Hewson and W. R. Ray, "The Effect of Electrostatic Screening of Rogowski Coils Designed for Wide-Bandwidth Current Measurement in Power Electronic Applications," in Proc. of the IEEE Power Electronics Specialists Conference (PESC), vol. 2, Jun. 2004, pp. 1143-1148.
[17] A. Küchler, Hochspannungstechnik: Grundlagen - Technologie - Anwendungen (in German). Springer, 2009.

[18] R. Bartnikas, Engineering Dielectrics: Measurement Techniques. Electrical Properties of Solid Insulating Materials. Vol. IIB. ASTM, 1987.

[19] A. Roberts, "Stress Grading for High Voltage Motor and Generator Coils,” IEEE Elect. Insul. Mag., vol. 11, no. 4, pp. 26-31, Jul. 1995.

[20] D. Rothmund, D. Bortis, and J. W. Kolar, "Accurate Transient Calorimetric Measurement of Soft-Switching Losses of 10kV SiC MOSFETs," in Proc. of the IEEE Power Electronics for Distributed Generation Systems Symp. (PEDG), Jun. 2016, pp. 1-10.

[21] T. Guillod, F. Krismer, R. Färber, C. M. Franck, and J. W. Kolar, "Protection of MV/LV Solid-State Transformers in the Distribution Grid," in Proc. of the Industrial Electronics Society Conf. (IECON), Nov. 2015, pp. 3531-3538.

[22] L. Dalessandro, F. da Silveira Cavalcante, and J. W. Kolar, "SelfCapacitance of High-Voltage Transformers," IEEE Trans. Power Electron., vol. 22, no. 5, pp. 2081-2092, Sep. 2007.

[23] Hitachi Metals, "FINEMET, Nanocrystalline Soft Magnetic Material," 2016. [Online]. Available: http://www.hitachi-metals.co.jp/products/ elec/tel/pdf/hl-fm9-h.pdf

[24] H. R. Carlon, Electrical Properties of Atmospheric Moist Air: A Systematic Experimental Study. U.S. Army Munitions/Chemical Command, 1988.

[25] C. Johansson and M. Robertsson, "Broadband Dielectric Characterization of a Silicone Elastomer," Journal of Electronic Materials, vol. 36 no. 9, pp. 1206-1210, 2007.

[26] M. Sato, A. Kumada, K. Hidaka, K. Yamashiro et al., "Dynamic Potential Distributions of Surface Discharge in Silicone Gel," IEEE Trans. Dielectr Electr. Insul., vol. 22, no. 3, pp. 1733-1738, Jun. 2015.

[27] R. Steiner, P. K. Steimer, F. Krismer, and J. W. Kolar, "Contactless Energy Transmission for an Isolated 100W Gate Driver Supply of a Medium Voltage Converter," in Proc. of the Industrial Electronics Society Conf. (IECON), Nov. 2009, pp. 302-307.

[28] F. P. Espino-Cortes, E. A. Cherney, and S. H. Jayaram, "Impact of Inverter Drives Employing Fast-Switching Devices on Form-Wound AC Machine Stator Coil Stress Grading," IEEE Elect. Insul. Mag., vol. 23, no. 1, pp. 16-28, Jan. 2007.

[29] M. Farahani, H. Borsi, E. Gockenbach, and M. Kaufhold, "Partial Discharge and Dissipation Factor Behavior of Model Insulating Systems for High Voltage Rotating Machines under Different Stresses," IEEE Elect. Insul. Mag., vol. 21, no. 5, pp. 5-19, Sep. 2005.

[30] F. Lü, D. Li, Y. Guo, C. Fu, and H. Wang, “Optimal Design of Compact Main Insulation Structure of PETT," in Proc. of the Electrical Machines and Systems Conf. (ICEMS), Oct. 2015, pp. 303-306.

[31] C. Zachariades, S. M. Rowland, I. Cotton, V. Peesapati, and D. Chambers, "Development of Electric-Field Stress Control Devices for a 132 kV Insulating Cross-Arm Using Finite-Element Analysis,” IEEE Trans. Power Del., vol. 31, no. 5, pp. 2105-2113, Oct. 2016.

[32] M. Steiner and H. Reinold, "Medium Frequency Topology in Railway Applications," in Proc. of the European Conf. Power Electronics and Applications (EPE), Sep. 2007, pp. 1-10.

[33] L. Paulsson, B. Ekehov, S. Halen, T. Larsson et al., "High-Frequency Impacts in a Converter-Based Back-to-Back Tie; The Eagle Pass Installation," IEEE Trans. Power Del., vol. 18, no. 4, pp. 1410-1415, Oct. 2003.

[34] T. Bengtsson, F. Dijkhuizen, L. Ming, F. Sahlen et al., "Repetitive Fast Voltage Stresses-Causes and Effects,” IEEE Elect. Insul. Mag., vol. 25, no. 4, pp. 26-39, Jul. 2009.

[35] ASTM International, Standard Test Methods for DC Resistance or Conductance of Insulating Materials. ASTM International, 2007.

[36] B. Cougo, A. Tüysüz, J. Mühlethaler, and J. W. Kolar, "Increase of Tape Wound Core Losses Due to Interlamination Short Circuits and Orthogonal Flux Components," in Proc. of the Industrial Electronics Society Conf. (IECON), Nov. 2011, pp. 1372-1377.

[37] Kontakt Chemie, “GRAPHIT 33, Technical Data Sheet,” 2013. [Online]. Available: http://www.crceurope.com/wwwcrc/tds/TKC3\%20Graphit33. pdf 